\title{
GEOMETRIC CONDITIONS FOR INTERPOLATION
}

\author{
C. L. BELNA', S. A. OBAID AND D. C. RUNG ${ }^{2}$
}

\begin{abstract}
Let $\left\{z_{n}\right\}$ be a sequence lying in either the upper half-plane or the unit disc in the complex plane. If $\left\{z_{n}\right\}$ is a separated sequence we give a simple geometric condition that implies the sequence is an interpolating sequence for the algebra of bounded holomorphic functions. This result contains most of the known results of this type.
\end{abstract}

0. Introduction. Let $\Delta$ denote the unit disc $|z|<1$ in the complex plane and $\partial \Delta$ its boundary. A sequence $\left\{z_{n}\right\}$ in $\Delta$ is an interpolating sequence for the algebra $H^{\infty}(\Delta)$ of bounded holomorphic functions in $\Delta$ if, for each bounded sequence of complex numbers $\left\{w_{n}\right\}$, there exists a function $f$ in $H^{\infty}(\Delta)$ such that $f\left(z_{n}\right)=w_{n}$ for all $n$. For $\left\{z_{n}\right\}$ to be interpolating it is necessary and sufficient that

$$
\inf _{\substack{k=1 \\ k \neq n}}^{\infty} x\left(z_{n}, z_{k}\right)>0
$$

where

$$
\chi(z, w) \equiv|(z-w) /(1-\bar{w} z)|
$$

is the pseudohyperbolic distance on $\Delta$. The necessity was established independently by L. Carleson [1], W. K. Hayman [5], and D. J. Newman [7]; the sufficiency was proved by Carleson [1]. A sequence $\left\{z_{n}\right\}$ that satisfies $(0.1)$ is said to be uniformly separated.

In practice, condition (0.1) is difficult to work with. Thus, several authors have given more accessible conditions for interpolation in the particular case that $\left\{z_{n}\right\}$ converges to a boundary point. See Garnett [3, pp. 284-301,438-439] for some of these results and a general treatment of interpolating sequences. Some involve a separation condition that is weaker than $(0.1)$. The sequence $\left\{z_{n}\right\}$ is called pairwise separated or just separated if it satisfies

$$
\inf _{n \neq m} \chi\left(z_{n}, z_{m}\right)>0
$$

Received by the editors August 19, 1982 and, in revised form, November 1, 1982.

1980 Mathematics Subject Classification. Primary 30E05; Secondary 30D55.

'The first author's research was partially supported by a National Science Foundation grant.

${ }^{2}$ The third author acknowledges partial support from a sabbatical leave from the Pennsylvania State University taken at the University of Hawaii-Manoa. 
D. H. Wortman [9] proved the following equivalence concerning sequences $\left\{z_{n}\right\}$ that lie on a convex boundary arc and satisfy

$$
\inf _{n} \chi\left(z_{n}, z_{n+1}\right)>0
$$

A sequence $\left\{z_{n}\right\}$ that satisfies (0.3) is said to be consecutively separated.

THEOREM A. Let $\Gamma$ be a convex curve in $\Delta \cup \partial \Delta$ with $\Gamma \cap \partial \Delta=\{1\}$ and $\Gamma$ tangent to $\partial \Delta$ at 1 . If $\left\{z_{n}\right\}$ is a sequence of points on $\Gamma \backslash\{1\}$ with $\left|z_{n}\right| \uparrow 1$, then $(0.1)$ is satisfied if and only if $\left\{z_{n}\right\}$ is consecutively separated.

Another characterization is due to M. L. Weiss [8]; this result contains the previous result of Wortman. (In his paper [8], Weiss was mainly concerned with sequences that lie on special curves in $\Delta$ that are tangent to $\partial \Delta$ at 1 . In his statement of the following theorem, he included the hypothesis that $\left\{z_{n}\right\} \rightarrow 1$ tangentially; however, this condition is readily seen to be extraneous.)

THEOREM B. If the sequence $\left\{z_{n}=r_{n} e^{i \theta_{n}}\right\}$ in $\Delta$ satisfies $r_{n} \uparrow 1$ and $\theta_{n} \downarrow 0$, then $\left\{z_{n}\right\}$ satifies $(0.1)$ if and only if $\left\{z_{n}\right\}$ is consecutively separated.

Recently, E. A. Gerber and M. L. Weiss [4, Theorem 4.6] established the following supplement to Theorem B.

THEOREM C. If the sequence $\left\{z_{n}=r_{n} e^{i \theta_{n}}=x_{n}+i y_{n}\right\}$ in $\Delta$ is consecutively separated with $\theta_{n} \downarrow 0$ and $x_{n} \uparrow 1$, then it is interpolating.

Our main result, Theorem 1, contains Theorems A, B and C. To begin, we define a

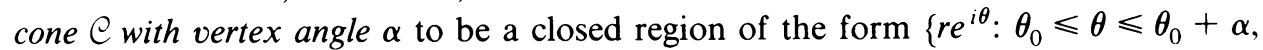
$r \geqslant 0\}(\pi / 2<\alpha<\pi)$. The rays $\left\{\theta=\theta_{0}\right\}$ and $\left\{\theta=\theta_{0}+\alpha\right\}$ are referred to, respectively, as the right and left boundary rays of $\mathcal{C}$. The cone that is the image of $\mathcal{C}$ under $T(z)=-z$ is denoted by $-\bigodot$, and the cone that is the image of $\bigodot$ under $T(z)=z+\zeta$ is denoted by $\mathcal{C}(\zeta)$. Also, the right and left boundary rays of $\mathcal{C}(\zeta)$ are the respective translated images of the right and left boundary rays of $C$.

We now state our main results. In $\$ 1$ we shall prove Theorem 1 , and in $\S 2$ we show that Corollary 1 follows readily from it. In the statements of our results, we shall allow $\left\{z_{n}\right\}$ to lie either in $\Delta$ or in the open upper half-plane $\mathcal{H}$. In $\mathcal{H}$ the pseudohyperbolic metric takes the form $\chi(z, w) \equiv|(z-w) /(z-\bar{w})|$, and the definition for the sequence to be interpolating is the same as that in $\Delta$ with $H^{\infty}(\Delta)$ replaced with $H^{\infty}(\mathcal{H})$.

THEOREM 1. Let $\left\{z_{n}\right\}$ be a separated sequence that lies in either $\Delta$ or $\mathcal{H}, \alpha$ a number strictly between $\pi / 2$ and $\pi$, and $N$ a nonnegative integer. If to each $n$ there corresponds a cone $\bigodot_{n}$ with vertex angle $\alpha$ such that

$$
z_{m} \in e_{n}\left(z_{n}\right) \text { for all but at most } N \text { indices } m>n,
$$

then $\left\{z_{n}\right\}$ is an interpolating sequence. 
COROLLARY 1 . Let $\left\{z_{n}\right\}$ be a consecutively separated sequence lying in either $\Delta$ or $\mathcal{H}$. If there exists a cone $e$ such that

$$
z_{n+1} \in \mathcal{C}\left(z_{n}\right) \text { for all } n,
$$

then $\left\{z_{n}\right\}$ is an interpolating sequence.

It is clear that Theorems A, B and C are immediate consequences of Corollary 1.

1. Proof of Theorem 1. Choose an even integer $J$ such that $2 \pi / J<(\pi-\alpha) / 2$, and let $e^{j}(j=1,2, \ldots, J)$ be all the cones with vertex angle $\pi-2 \pi / J$ formed from the rays $\{\theta=2 \pi j / J\}(j=1,2, \ldots, J)$. Then clearly each cone $\bigodot_{n}$ is contained in one of the cones $e^{j}$. Therefore, since the finite union of interpolating sequences is also interpolating, provided the union is a separated sequence, it suffices to prove Theorem 1 with hypothesis $(0.4)$ replaced with the hypothesis that there exists a fixed cone $e$ with vertex angle $\alpha$ such that

$$
z_{m} \in \mathcal{C}\left(z_{n}\right) \text { for all but at most } N \text { indices } m>n
$$

We prove Theorem 1 first for $\left\{z_{n}\right\}$ in $\mathcal{H}$. For $z, w \in \mathcal{H}$,

$$
\frac{\chi(z, w)}{1+\chi(z, w)} \leqslant \frac{|z-w|}{2 \operatorname{Im}(z)} \leqslant \frac{\chi(z, w)}{1-\chi(z, w)} .
$$

To see this, note that

$$
\frac{|z-w|}{2 \operatorname{Im}(z)}=\frac{|z-w|}{|(z-\bar{w}+\bar{w}-\bar{z})|}=\frac{|(z-w) /(z-\bar{w})|}{|1+(\bar{w}-\bar{z}) /(z-\bar{w})|},
$$

and then apply the triangle inequalities to the denominator of the last term. By the left inequality in (1.2) and the fact that $\left\{z_{n}\right\}$ is separated, there exists a constant $A$ such that

$$
\operatorname{Im}\left(z_{n}\right) \leqslant A\left|z_{n}-z_{m}\right| \text { for all } n \text { and } m .
$$

We shall make use of the inequality later in the proof.

According to J. Garnett [2] (see also [6, pp. 280-282]), to prove that the separated sequence $\left\{z_{n}\right\}$ is interpolating it is sufficient to show that it generates a Carleson measure, that is, to show that there exists a constant $K$ such that $\Sigma_{z_{n} \in \delta} \operatorname{Im}\left(z_{n}\right)<K d$ for each square $\delta$ of the form $\left\{x+i y: x_{0} \leqslant x \leqslant x_{0}+d, 0 \leqslant y \leqslant d\right\}$.

Consider an arbitrary square $\mathcal{S}=\left\{x+i y: x_{0} \leqslant x \leqslant x_{0}+d, 0 \leqslant y \leqslant d\right\}$. We may assume that the vertex angle $\alpha$ of $\mathcal{C}$ is close enough to $\pi$ so that $\delta \subset \mathcal{C}(z)$ for one of the corners $z$ of $\mathcal{S}$. We give the proofs for $z=x_{0}+i d$; the other three proofs are similar. See Figure 1.

Let 2 denote the parallelogram obtained by intersecting $e\left(x_{0}+i d\right)$ and $-\mathcal{C}\left(x_{0}+d\right)$. Clearly, $\delta \subset \mathcal{2}$, and to complete the proof we need only show that there exists a constant $K$, independent of $x_{0}$ and $d$, such that

$$
\sum_{z_{n} \in \mathcal{Q}} \operatorname{Im}\left(z_{n}\right) \leqslant K d \text {. }
$$




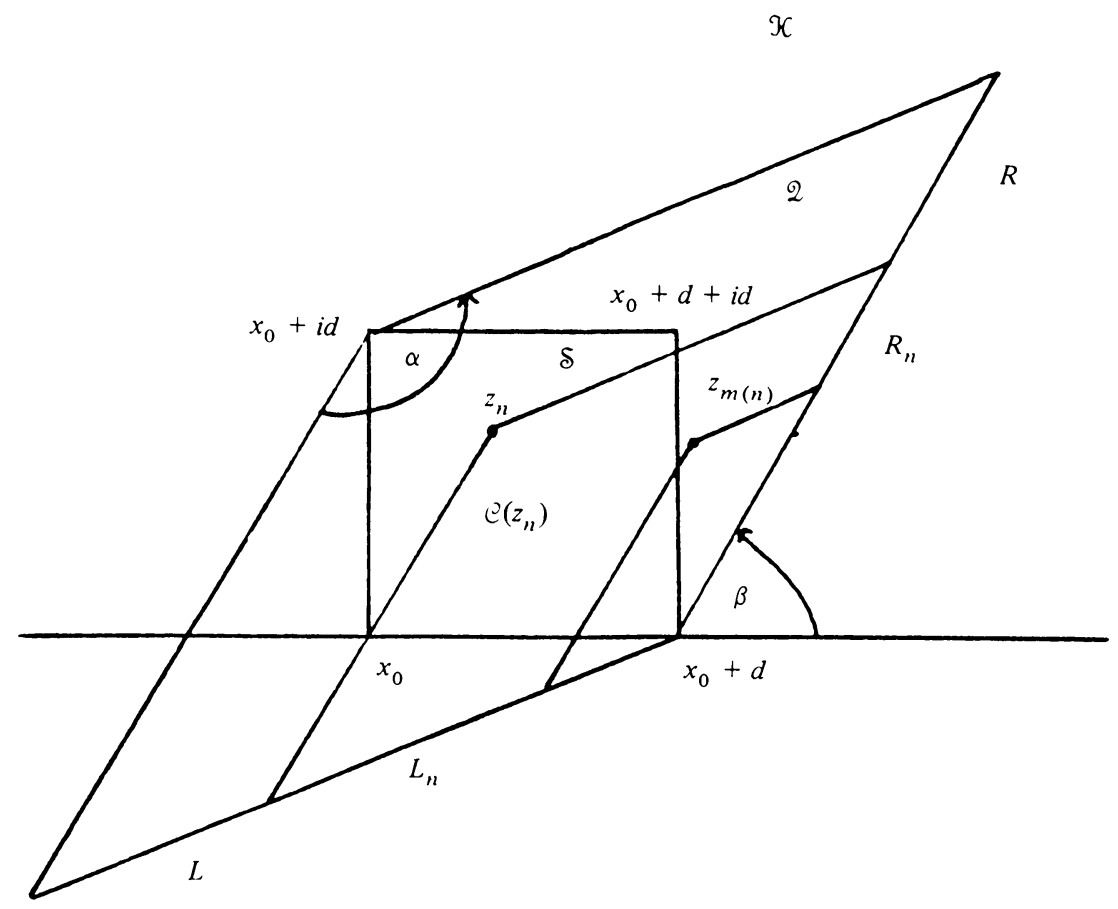

FIGURE 1

Let $R$ and $L$ denote the sides of 2 corresponding to the right and left boundary rays of $-\mathcal{C}\left(x_{0}+d\right)$, and let $|R|$ and $|L|$ denote their lengths. If $\beta$ is the angle that $R$ makes with the positive real axis, then two applications of the law of sines yield

$$
|R|+|L|=\sqrt{2} d(\csc \alpha)[\sin (\alpha+\beta-3 \pi / 4)+\sin (3 \pi / 4-\beta)] \equiv B d
$$

Note that

$$
\operatorname{Im}(z)<|R|+|L| \text { for each } z \in \mathcal{Q} \text {. }
$$

We suppose there are points $z_{n} \in \mathcal{2}$; otherwise (1.4) holds vacuously. Separate that portion of $\left\{z_{n}\right\}$ in 2 into two sets $P$ and $T$ with $z_{n} \in P$ if $\mathcal{C}\left(z_{n}\right)$ contains at least one $z_{m} \in \mathcal{Q}$ with $m>n$; otherwise $z_{n} \in T$. First we show that $T$ contains at most $N+1$ points. Let $z_{k}$ be the term in $T$ of least index. Because $z_{k}$ is the only point of $T$ in $C\left(z_{k}\right),(1.1)$ implies that $T$ has at most $N+1$ points. Combining (1.5) and (1.6) we have

$$
\sum_{z_{n} \in T} \operatorname{Im}\left(z_{n}\right)<(N+1) B d
$$


For $z_{n} \in P$, let $m(n)$ be the smallest $m>n$ such that $z_{m} \in \mathcal{E}\left(z_{n}\right) \cap \stackrel{0}{*}$. Then let $R_{n}$ and $L_{n}$ denote the intersection of $己\left(z_{n}\right) \backslash \mathfrak{E}\left(z_{m(n)}\right)$ with $R$ and $L$, respectively. (See Figure 1.) Note that if $z_{m(n)} \in \partial \stackrel{c}{ }\left(z_{n}\right)$, then one of $R_{n}$ or $L_{n}$ equals $\varnothing$. In any case it is easy to see that $\left|z_{n}-z_{m(n)}\right| \leqslant\left|R_{n}\right|+\left|L_{n}\right|$, and by (1.3) we have

$$
A^{-1} \sum_{z_{n} \in P} \operatorname{Im}\left(z_{n}\right) \leqslant \sum_{z_{n} \in P}\left(\left|R_{n}\right|+\left|L_{n}\right|\right) \text {. }
$$

We claim that no $z \in L$ is contained in more than $2 N$ of the segments $L_{n}$. Fix $z \in L$ and let $n^{*}$ be the smallest $n$ such that $z \in L_{n}$. If $m \geqslant m\left(n^{*}\right)$ and $z_{m} \in \mathcal{E}^{2}\left(z_{m\left(n^{*}\right)}\right)$, then $z \notin L_{m}$. Consequently, (1.1) implies there are at most $N$ indices $m \geqslant m\left(n^{*}\right)$ for which $z \in L_{m}$. By the same hypothesis $m\left(n^{*}\right)-n^{*} \leqslant N+1$. This establishes the claim and, hence, it follows that

$$
\sum_{z_{n} \in P}\left|L_{n}\right| \leqslant(2 N)|L|
$$

A similar argument shows that

$$
\sum_{z_{n} \in P}\left|R_{n}\right| \leqslant(2 N)|R|
$$

Using inequalities (1.8)-(1.10) together with (1.5) we obtain

$$
\sum_{z_{n} \in P} \operatorname{Im}\left(z_{n}\right) \leqslant 2 N A B d .
$$

In view of (1.7) and (1.11) we see that (1.4) holds for $K$ equal to the sum of $2 N A B$ and $(N+1) B$. This completes the proof of Theorem 1 for $\left\{z_{n}\right\}$ in $\mathcal{K}$.

We now prove Theorem 1 for $\left\{z_{n}\right\}$ in $\Delta$. Because $z_{m} \in \mathcal{E}\left(z_{n}\right)$ implies $\mathcal{E}\left(z_{m}\right) \subset$ $\mathcal{E}\left(z_{n}\right)$, a simple geometric argument can be used to deduce that $\left\{z_{n}\right\}$ converges to a point $\zeta \in \partial \Delta$. Using a rotation if necessary, we may assume that $\zeta=1$. Then, since $1 \in \mathcal{C}^{2}\left(z_{n}\right)$ for each $n$ and since $\alpha<\pi$, there is a $\tau \in \partial \Delta$ with $\tau \notin \mathcal{C}\left(z_{n}\right)$ for $n$ large; hence we may assume that $\tau$ belongs to none of the cones $e\left(z_{n}\right)$.

Consider the conformal mapping

$$
T(z)=e^{i \theta}(z-1) /(z-\tau)
$$

of $\Delta$ onto $\mathcal{H}$. Then $\left\{T\left(z_{n}\right)\right\}$ is a separated sequence in $\mathcal{H}$ that converges to 0 . Since $T(\tau)=\infty$, the image of $\mathcal{C}\left(z_{n}\right) \cap \Delta$ is the intersection with $\mathcal{H}$ of the interiors of two bounded circles that intersect at $T\left(z_{n}\right)$ and $T(\infty)$. Thus if $\bigodot_{n}\left(T\left(z_{n}\right)\right)$ is the cone that contains 0 and is formed by the tangents to the intersecting circles at $T\left(z_{n}\right)$, then this cone has a vertex angle $\alpha$ and $T\left(z_{m}\right) \in \bigodot_{n}\left(T\left(z_{n}\right)\right)$ for all but at most $N$ indices $m>n$. Therefore, by Theorem 1 for sequences in $\mathcal{H}$, the sequence $\left\{T\left(z_{n}\right)\right\}$ is an interpolating sequence in $\mathcal{H}$. This clearly implies that $\left\{z_{n}\right\}$ is an interpolating sequence in $\Delta$, and the proof of Theorem 1 is complete.

2. Proof of Corollary 1 . Suppose $\left\{z_{n}\right\}$ is a consecutively separated sequence in $\mathcal{H}$ and suppose condition (0.5) is satisfied for some cone $\mathcal{C}$. Then a basic geometric argument will show that the distance between $z_{n}$ and any point in $\mathcal{C}\left(z_{n+1}\right)$ is at least $\left|z_{n}-z_{n+1}\right| \sin \alpha$, where $\alpha$ is the vertex angle of $C$. Thus, for any $m>n$ we have

$$
\left|z_{n}-z_{m}\right| \geqslant\left|z_{n}-z_{n+1}\right| \sin \alpha \text {. }
$$


The left inequality in (1.2) for $m=n+1$ gives $\left|z_{n}-z_{n+1}\right| / y_{n} \geqslant \chi\left(z_{n}, z_{n+1}\right)$, where $y_{n}=\operatorname{Im}\left(z_{n}\right)$. Combining this inequality with (2.1) and using the hypothesis that $\left\{z_{n}\right\}$ is consecutively separated, we obtain a constant $Q$ such that

$$
\left|z_{n}-z_{m}\right| / y_{n}>Q \text { for } m \neq n \text {. }
$$

Now we deduce from the right inequality in (1.2) that $\chi\left(z_{n}, z_{m}\right)>Q /(Q+2)$; that is, $\left\{z_{n}\right\}$ is separated, and it follows from Theorem 1 that $\left\{z_{n}\right\}$ is interpolating.

The proof for $\left\{z_{n}\right\}$ in $\Delta$ is identical once we obtain the disc analogue of (1.2): for $z, w \in \Delta$,

$$
\frac{\chi(z, w)}{1+|z| \chi(z, w)} \leqslant \frac{|z-w|}{1-|z|^{2}} \leqslant \frac{\chi(z, w)}{1-|z| \chi(z, w)} .
$$

The proof is entirely similar to that of (1.2) in that

$$
\frac{|z-w|}{1-|z|^{2}}=\frac{|z-w|}{|1-z \bar{w}+z \bar{w}-z \bar{z}|}=\frac{|(z-w) /(1-z \bar{w})|}{|1+z((\bar{w}-\bar{z}) /(1-z \bar{w}))|} .
$$

The triangle inequalities applied to the denominator of the last term give (2.2).

3. Sharpness of Theorems 1 and C. Let $p$ be a positive integer and consider the square

$$
\varsigma_{p} \equiv\left\{x+i y: 0 \leqslant x \leqslant 2^{-p^{2}}, 0 \leqslant y \leqslant 2^{-p^{2}}\right\} .
$$

Within $\varsigma_{P}$ consider the set of points

$$
\mathfrak{s}_{p} \equiv\left\{(\sigma+i) 2^{-p^{2}-\lambda}: \lambda=0,1, \ldots, p-1 ; \sigma=1,2, \ldots, 2^{\lambda}\right\},
$$

and set $\mathfrak{s}=\cup_{\mathfrak{S}_{p}}$. Then the following facts are easily proven:

(I) $\sum_{z \in_{\mathfrak{s}} p} \operatorname{Im}(z)=p 2^{-p^{2}}$;

(II) $\chi(z, w) \geqslant \frac{1}{3}$ for any distinct points $z, w \in \mathfrak{s}$; and

(III) $\mathfrak{I}_{p} \subset\left\{\left|z-i a_{p}\right| \leqslant a_{p}\right\}$ for $a_{p}=2^{-p^{2}+p-2}+2^{-p^{2}-p}$.

Thus, by ordering $\mathfrak{g}$ from "top down and left to right", we obtain the following example.

EXAMPLE. There exists a noninterpolating, separated Blaschke sequence $\left\{z_{n}\right\} \subset \mathbb{H}$ such that $\left\{z_{n}\right\}$ is eventually in every disc in $\mathcal{H}$ that is tangent to $\partial \mathcal{H}$ at 0 and to each $z_{n}$ there corresponds a cone $\mathcal{C}_{n}$ in the complement of $\mathcal{H}$ with the property that $z_{m} \in \mathcal{C}_{n}\left(z_{n}\right)$ for each $m>n$. (Note that each $\bigcup_{n}$ has vertex angle $\alpha_{n}<\pi$, but $\sup _{n} \alpha_{n}=\pi$.)

\section{REFERENCES}

1. L. Carleson, An interpolation problem for bounded analytic functions, Amer. J. Math. 80 (1958), $921-930$.

2. J. Garnett, Interpolating sequences for bounded harmonic functions, Indiana Univ. Math. J. 21 (1971), $187-192$.

3. B__ Bounded analytic functions, Academic Press, New York, 1981.

4. E. A. Gerber and M. L. Weiss, Interpolation, trivial and non-trivial homomorphisms in $H^{\infty}$, J. Math. Soc. Japan 34 (1982), 173-185.

5. W. K. Hayman, Interpolation by bounded functions, Ann. Inst. Fourier (Grenoble) 8 (1959), 277-290.

6. P. Koosis, Introduction to $H^{p}$ spaces, London Math. Soc. Lecture Note Ser. (2), Vol. 40, Cambridge Univ. Press, Cambridge, 1980.

7. D. J. Newman, Interpolation in $H^{\infty}$, Trans. Amer. Math. Soc. 92 (1959), 501-507. 
8. M. L. Weiss, Some $H^{\infty}$-interpolating sequences and the behavior of certain of their Blaschke products, Trans. Amer. Math. Soc. 209 (1975), 211-223.

9. D. H. Wortman, Interpolating sequences on convex curves in the open unit disc, Proc. Amer. Math. Soc. 48 (1975), 157-164.

Syracuse Research Corporation, Syracuse, New York 13210

Department of Mathematics, University of TeXas, San Antonio, Texas 78229

Department of Mathematics, Pennsylvania State University, University Park, Pennsylvania 16802 